\title{
Muon telescope based on Micromegas detectors: From design to data acquisition
}

Ignacio Lázaro $^{1, a}$, Fanny Hivert ${ }^{1,2}$, Jean-Baptiste Decitre ${ }^{1}$, Rui De Oliveira ${ }^{3}$, Olivier Pizzirusso ${ }^{3}$, Hans Muller $^{3}$, Fabien Jeanneau ${ }^{4}$, Ioannis Giomataris ${ }^{4}$ and Stéphane Gaffet ${ }^{1,5}$

${ }^{1}$ LSBB, U. of Nice, U. of Avignon, CNRS, Aix-Marseille U., OCA, Rustrel, France

${ }^{2}$ Fondation MAIF, Paris, France

${ }^{3}$ CERN, Meyrin, Swiss

${ }^{4}$ CEAIRFU, Saclay, France

${ }^{5}$ GEOAZUR, University of Nice, CNRS, IRD, OCA, Sophia-Antipolis, France

\begin{abstract}
We describe the basis of the muon telescope used within the Temporal Tomography Densitometric by the Measure of Muons (T2DM2) project developed in the LSBB URL facilities. The telescope allows measuring the flux of muons, as well as their energy and origin for the characterization of spatial and temporal rock density variations.
\end{abstract}

\section{Bulk Micromegas}

The Micromegas is a micro-pattern gas detector first introduced in 1996 [1], which detects particles by amplifying the charges created by the ionization of the gas. As shown in Fig. 1, it is a two-stage parallel plate avalanche chamber with a narrow amplification gap. Regularly-spaced insulating supports keep constant the distance between the mesh and the electrode (the resistive tracks). As a consequence, there is a dead area of $0.4 \%$ due to the space occupied by the pillars (Fig. 2R). The more recent bulk Micromegas [2] includes resistive tracks acting as spark protection to reduce the discharge current by a factor of 1000, preventing the front end electronics from being damaged [3]. Underneath, the readout tracks are distributed into two superimposed layers separated by an insulator, with the tracks arranged along the $\mathrm{X}$ and $\mathrm{Y}$ axes respectively.

The dimensions of the Printed Circuit Board (PCB), where the detector is mounted, are $119.5 \mathrm{~cm} \times$ $61 \mathrm{~cm}$. Nevertheless, the active surface covered by the readout tracks is $0.45 \mathrm{~m}^{2}$ due to the space reserved for assembly and instrumentation, giving a pixel density of more than 116 pixels per $\mathrm{cm}^{2}$.

The telescope has exceptional spatial and multi-track resolutions of approximately $12 \mu \mathrm{m}$ and $500 \mu \mathrm{m}$ respectively, angular resolution of $0.3 \mathrm{mrad}$, and time resolution of order few ns [4]. The efficiency depends on several factors such as the gas employed, the electric field and the rate speed, but it can go up to $98 \%$.

\footnotetext{
${ }^{a}$ Corresponding author: ignacio. laroc@lsbb.eu
}

This is an Open Access article distributed under the terms of the Creative Commons Attribution License 4.0, which permits unrestricted use, distribution, and reproduction in any medium, provided the original work is properly cited. 


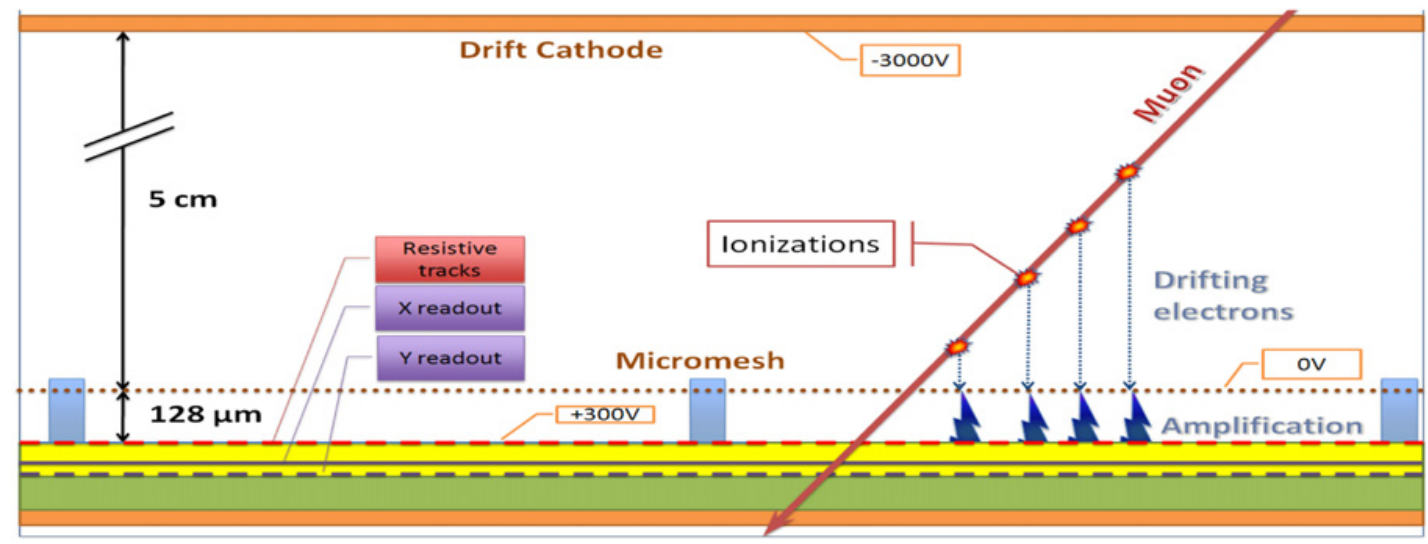

Figure 1. Schematic section of the muon telescope showing the gas ionization and signal collection.
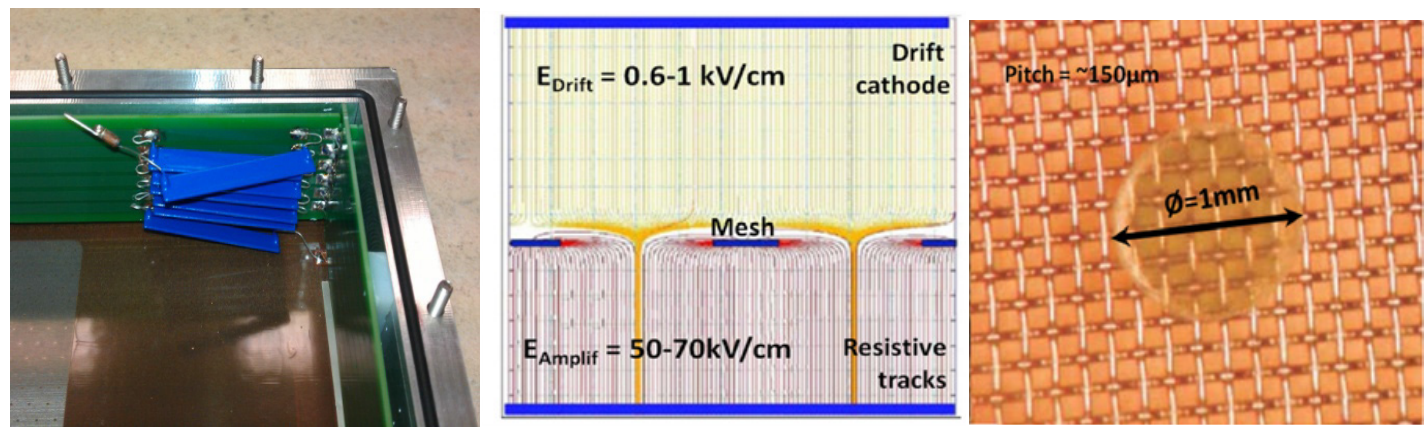

Figure 2. Left: detail of the detector, aluminium frame and the PCB for electric field homogenization; Centre: simulation of the electric field isolines [5]; Right: zoom of the micromesh support [6].

\section{Electric field configuration}

The Time Projection Chamber (TPC) is a position-sensitive electron collection system. It has a gas volume divided in two regions by a woven stainless steel wire micromesh: the conversion and drift volume, and the amplification gap.

The conversion and drift volume comprises the $5 \mathrm{~cm}$ of space between the drift cathode and the micromesh, which is 10-15 times larger than the most common sizes in similar devices. Inside the frame and parallel to it, there is a printed circuit board to distribute the electric field (Fig. 2L). It links the drift cathode to the ground through six copper tracks connected by resistors; creating a homogeneous field on the order of $0.6-1 \mathrm{kV} / \mathrm{cm}$. If no electric field were applied, the ion/electron pair generated by the ionization of the gas due to the passage of the muon would recombine without generating a signal. But within an electric field, the electrons (in the right in Fig. 1) will drift towards the amplification electrode (the resistive tracks).

When an electron arrives to the mesh, it enters in the amplification gap of $128 \mu \mathrm{m}$, with an electric field of typically $50-70 \mathrm{kV} / \mathrm{cm}$. The electric field in both regions is homogeneous, but it has a microscopic transition zone nearby the mesh. The field lines are compressed near the openings of the micromesh with a funnel-like shape, as shown in Fig. 2C. According to the simulations, the compaction of the isofield lines depends on the electric ratio between electric fields in the two volumes [5]. 


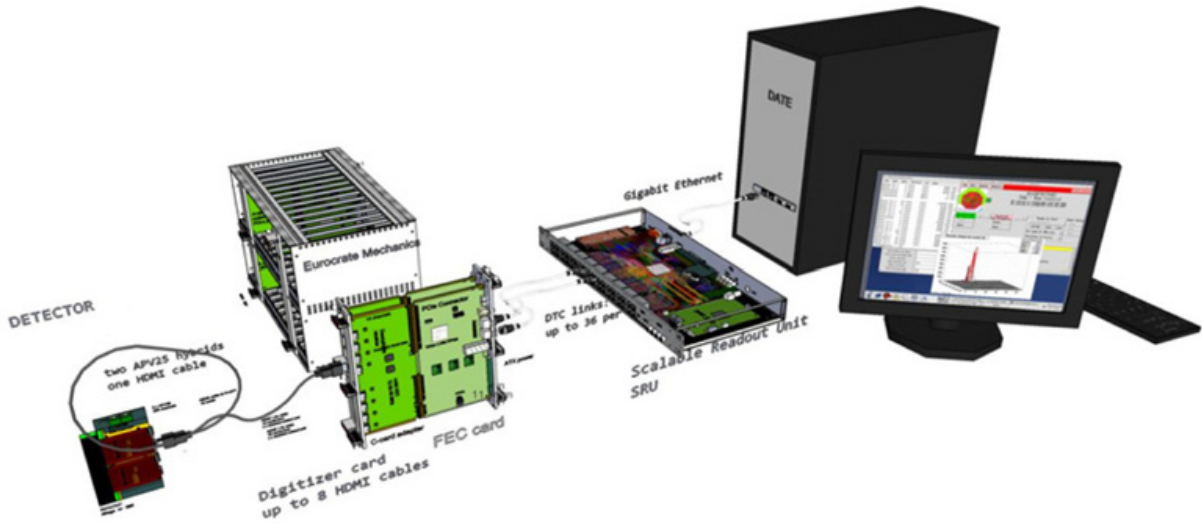

Figure 3. Elements of the Scalable Readout System.

Accelerated by the field, the electron reaches enough energy to ionize the gas, which multiplies the primary charge to create a significant signal. The signal so generated is collected by resistive tracks, which induces a true signal in the 2-axis readout tracks. This allows counting particles, track their trajectory and determine their energy.

\section{Instrumentation}

The elements for data acquisition (see Fig. 3) come from CERN's RD51 collaboration, which started in 2009 to develop a Scalable Readout System (SRS) in order to enable a portable multichannel readout system for micropattern gas detectors [7].

Each muon telescope has 1024 and 512 channels respectively in $\mathrm{X}$ and $\mathrm{Y}$ axis coming from the readout tracks. They are grouped into 128 channels nodes, which make a total of 12 per detector. In each of these nodes, there is an APV25 front end card (see Fig. 4L) which contains an analog preamplifier and shaper for every channel. These cards usually operate in pairs master/slave; the slave being attached to the master and the master connected to the digitizer card (ADC) via HDMI cables. The analog output signals are transmitted via a chip link up to $30 \mathrm{~m}$ away.

The ADC card has 8 inputs (up to 16 APV hybrids), of which 6 are used with one telescope and 2 reserved as spare. It has a 12-bit octal-ADC chips with up to $50 \mathrm{MS} / \mathrm{s}$ sampling rate and programmable gains. The ADC digitizes the data stream for FPGA processing in the Front End Concentrator (FEC). Both cards are electrically and mechanically connected via PCIe connectors that provide Gb speed. Thus, every telescope needs an ADC coupled to a FEC, called SRS combo.

The Scalable Readout Unit (SRU) multiplexes data for up to 36 SRS combo, with a maximum of $80 \mathrm{kchannels,}$ whose output is a Gigabit Ethernet cable. Finally, the PC contains all the software needed to monitor pedestals and noise, and analyze the physics data.

There are different ways to trigger the data acquisition with both internal and external signals. Selecting the signal from the micromesh allows the detector to run in a self-triggered mode. This signal, once amplified and processed in the NIM rack (Fig. 4R), is sent to the FEC card indicating the events. On the other hand, the trigger can be originated in a different detector to study coincidences, among others.

It is important to anticipate the muon flux to assess telescope's performance. Simulations have been made to predict the muon flux variation with depth, paying particular attention on muon scattering inside the rock [9]. 

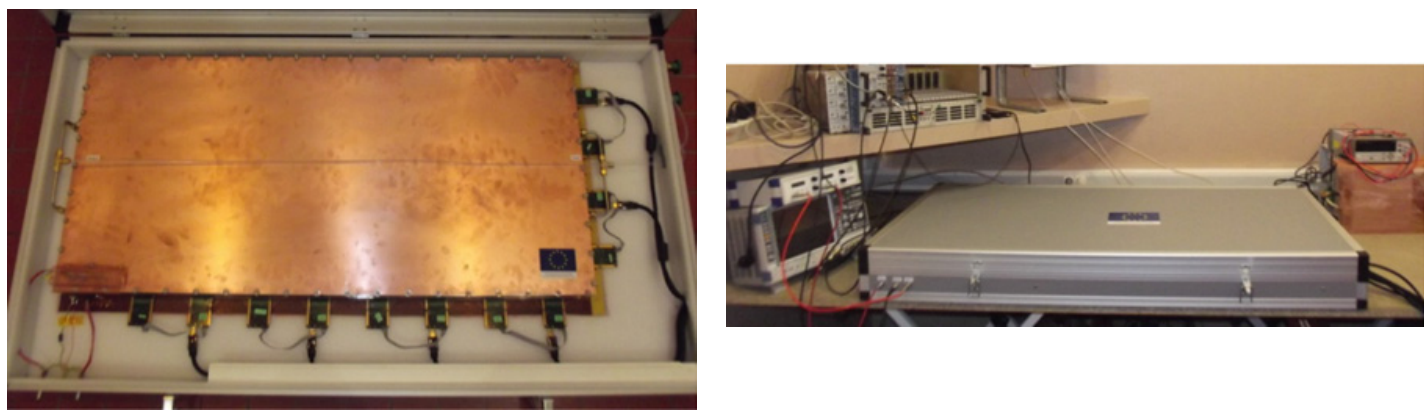

Figure 4. Left: muon telescope inside its case. Right: telescope, its instrumentation and gas conditioner.

\section{Summary and outlook}

Muon tomography based on the measurement of cosmic ray muon attenuation or absorption, is a rising technique with promising applications such as characterization of spatial and temporal rock density variations.

The ongoing technology transfer of the bulk Micromegas technology, soon suitable for industrial production, will make this kind of detector very popular. The low fabrication cost and robustness of the electrode materials makes it an excellent candidate for diverse purposes, such as astrophysics or geophysics.

The main advantages of the scalable readout system chosen to instrument the telescope is the high scalability of the number of channels and telescopes in the network, the portability and relatively inexpensiveness ( $<2 € /$ channel). In addition, the modular design allows enclosing and tracking hardware errors in the system and a quick replacement of inoperative parts.

The first phase of the T2DM2 project aims at deploying a network of six muon telescopes, as a methodological demonstrator, within the low background inter-Disciplinary Underground Science \& Technology (LSBB) before its future operation in other works and underground spaces and monitoring of unstable cliffs and gravitational movements.

This work is supported by the European Fund for Regional Development (FEDER), the platform for fundamental \& applied low background inter-Disciplinary Underground Science \& Technology (LSBB) and the MAIF foundation.

\section{References}

[1] Y. Giomataris et al., MICROMEGAS: a high-granularity position-sensitive gaseous detector for high particle-flux environments. Nucl. Instr. Meth. A376 (1996) 29-35.

[2] Y. Giomataris et al., Micromegas in a Bulk. doi:10.1016/j.nima.2005.12.222.

[3] R. Oliveira et al., First tests of "bulk" MICROMEGAS with resistive cathode mesh. arXiv:1007.0211v1 (2010).

[4] Y. Giomataris, MICROMEGAS: results and prospects. ICFA Instrumentation Bulletin (1999).

[5] M. Titov, Perspectives of Micro-Pattern Gaseous Detector Technologies for Future Physics Projects. arXiv:1308.3047 (2013).

[6] S. Duarte Pinto, Micropattern gas detector technologies and applications. The work of the RD51 collaboration. doi:10.1109/NSSMIC.2010.5873870 (2010). 
[7] S. Martoiu et al., Development of the scalable readout system for micro-pattern gas detectors and other applications. doi:10.1088/1748-0221/8/03/C03015 (2013).

[8] K. Gnanvo et al., Detection and Imaging of High-Z Materials with a Muon Tomography Station Using GEM Detectors. doi:10.1109/NSSMIC.2010.5873822.

[9] F. Hivert et al., Muon tomography of rock density using Micromegas - TPC telescope. i-Dust conference (2014) Pending publication. 